\title{
Effect of oxymatrine on liver gluconeogenesis is associated with the regulation of PEPCK and G6Pase expression and AKT phosphorylation
}

\author{
YU-XIAN ZHU ${ }^{1,2 *}$, HAI-QING HU ${ }^{1 *}$, MEI-LING ZUO ${ }^{1}$, LI MAO $^{3}$, GUI-LIN SONG ${ }^{1,4}$, \\ TAO-MING LI ${ }^{1}$, LI-CHEN DONG ${ }^{1}$, ZHONG-BAO YANG $^{1,4}$ and MD SAYED ALI SHEIKH ${ }^{5}$ \\ ${ }^{1}$ The Affiliated Changsha Hospital of Hunan Normal University, Changsha, Hunan 410006; \\ ${ }^{2}$ College of Medicine, Hunan Normal University Changsha, Hunan 410000; \\ ${ }^{3}$ Department of Basic Medicine, Changsha Health Vocational College, Changsha, Hunan 410600; \\ ${ }^{4}$ Institute of Emergency and Critical Care Medicine of Changsha, Changsha, Hunan 410006, P.R. China; \\ ${ }^{5}$ Internal Medicine Department, Cardiology, College of Medicine, Al Jouf University, Sakaka, Al Jouf 72388, Saudi Arabia
}

Received September 21, 2020; Accepted March 5, 2021

DOI: $10.3892 /$ br.2021.1432

\begin{abstract}
An increase in liver gluconeogenesis is an important pathological phenomenon in type 2 diabetes mellitus (T2DM) and oxymatrine is an effective natural drug used for T2DM treatment. The present study aimed to explore the effect of oxymatrine on gluconeogenesis and elucidate the underlying mechanism. Male Sprague-Dawley rats were treated with a high-fat diet and streptozotocin for 4 weeks to induce T2DM, and HepG2 cells were treated with $55 \mathrm{mM}$ glucose to simulate T2DM in vitro. T2DM rats were treated with oxymatrine (10 or $20 \mathrm{mg} / \mathrm{kg}$ weight) or metformin for 4 weeks, and HepG 2 cells were treated with oxymatrine $(0.1$ or $1 \mu \mathrm{M})$, metformin $(0.1 \mu \mathrm{M})$, or oxymatrine combined with MK-2206 (AKT inhibitor) for $24 \mathrm{~h}$. Fasting blood glucose and insulin sensitivity of rats were measured to evaluate insulin resistance. Glucose production and uptake ability were measured to evaluate gluconeogenesis in HepG2 cells, and the expression of related genes was detected to explore the molecular mechanism. Additionally, the body weight, liver weight and liver index were measured and hematoxylin and eosin staining was performed to evaluate the effects of the disease. The fasting glucose levels of T2DM rats was $16.5 \mathrm{mmol} / \mathrm{l}$, whereas in the control rats, it was $6.1 \mathrm{mmol} / \mathrm{l}$. Decreased insulin sensitivity (K-value, 0.2 ), body weight loss (weight,
\end{abstract}

Correspondence to: Dr Zhong-Bao Yang, The Affiliated Changsha Hospital of Hunan Normal University, 70 Lu-Shan Road, Changsha, Hunan 410006, P.R. China

E-mail:yzb55@yahoo.com

*Contributed equally

Key words: type 2 diabetes mellitus, gluconeogenesis, AKT, phosphoenolpyruvate carboxykinase, glucose-6-phosphatase, oxymatrine
$300 \mathrm{~g}$ ), liver weight gain, liver index increase (value, 48) and morphological changes were observed in T2DM rats, accompanied by reduced AKT phosphorylation, and upregulated expression of phosphoenolpyruvate carboxykinase (PEPCK) and glucose-6-phosphatase (G6Pase). High-glucose treatment significantly increased glucose production and decreased glucose uptake in HepG2 cells, concomitant with a decrease in AKT phosphorylation and increase of PEPCK and G6Pase expression. In vivo, oxymatrine dose-dependently increased the sensitivity of T2DM rats to insulin, increased AKT phosphorylation and decreased PEPCK and G6Pase expression in the liver, and reversed the liver morphological changes. In vitro, oxymatrine dose-dependently increased AKT phosphorylation and glucose uptake of HepG2 cells subjected to high-glucose treatment, which was accompanied by inhibition of the expression of the gluconeogenesis-related genes, PEPCK and G6Pase. MK-2206 significantly inhibited the protective effects of oxymatrine in high-glucose-treated cells. These data indicated that oxymatrine can effectively prevent insulin resistance and gluconeogenesis, and its mechanism may be at least partly associated with the regulation of PEPCK and G6Pase expression and AKT phosphorylation in the liver.

\section{Introduction}

Diabetes mellitus (DM) is a common chronic metabolic disease, which is primarily characterized by an increase in blood glucose levels and its complications are usually caused by insulin resistance or deficiency (1). In China, $11 \%$ of the population are diagnosed with DM at present, and the age of onset has been decreasing in recent years (2). As the most common type of diabetes, type 2 DM (T2DM) accounts for $90-95 \%$ of all DM cases (3). It has been confirmed that abnormal glucose metabolism in the liver is one of the primary pathological factors of T2DM, and diabetic patients often exhibit disrupted glycogenesis and glycogenolysis, with the role of glycogenesis being particularly important (4). In the process of gluconeogenesis, phosphoenolpyruvate carboxylase 
(PEPCK) and glucose-6-phosphatase (G6Pase) are the key enzymes in the liver regulating the conversion of non-sugar substances into glucose. The increase in their expression is associated with the enhancement of gluconeogenesis (5). Therefore, PEPCK and G6Pase are important targets for diabetes treatment and development of novel drugs.

The insulin signaling pathway serves an important role in T2DM. Normal insulin signaling can inhibit the occurrence of gluconeogenesis in hepatocytes, reduce the output of glucose, and increase the utilization of glucose by peripheral tissues (6). AKT is a key downstream molecule in the insulin signaling pathway, and its abnormal phosphorylation is closely associated with insulin resistance and gluconeogenesis, which is considered as a marker of T2DM (7). Therefore, screening drugs that target AKT based on traditional Chinese herbal medicines that exhibit anti-gluconeogenic or anti-insulin resistance properties may be useful for the treatment of diabetes.

Oxymatrine is an important active component of the traditional Chinese herbal medicine Sophora flavescens Ait. It has a variety of pharmacological effects, such as anti-inflammatory, antiallergic, antiviral, antifibrotic, blood sugar- and blood lipid-lowering properties (8-13). It was previously demonstrated that oxymatrine has anti-T2DM properties and may be used for obesity-related diseases as an adjuvant therapy $(14,15)$. Its mechanism is associated with the regulation of the PI3K/AKT pathway (15); however, the exact mechanism remains to be elucidated. It was demonstrated that oxymatrine can effectively improve the sensitivity of T2DM rats to insulin, indicating that oxymatrine may be a new therapeutic strategy for anti-gluconeogenesis in patients with T2DM (16). However, few studies to date have explored whether the protective mechanism of oxymatrine on diabetes is associated with the phosphorylation of AKT and the regulation of the expression of gluconeogenesis-related genes.

The aim of the present study was to explore the effect of oxymatrine on gluconeogenesis in T2DM rats, and to determine whether its mechanism of action is associated with the regulation of PEPCK and G6Pase expression, as well as AKT phosphorylation in the liver.

\section{Materials and methods}

Animal experiments. A total of 40 male Sprague-Dawley rats ( 8 weeks old; weight, $270 \pm 20 \mathrm{~g}$ ) were provided by Hunan SJA Laboratory Animal Co.,Ltd. All rats were raised according to the standard conditions: 12 -h light/dark cycle, $25^{\circ} \mathrm{C}$ ambient temperature and $60 \%$ humidity. All rats were provided ad libitum access to food and water. The study was performed in accordance with the National Research Council (US) Committee guidelines for the care and use of experimental animals (16), and was approved by the Veterinary Animal Care and use Committee of Hunan Normal University (approval no. 2019111).

The T2DM rat model was established as described by Ma et al (17) with some modifications: The rats were fed a high-fat diet from the beginning of the experiments, and were administered streptozotocin by intraperitoneal injection (from week 1 to 4) every 2 days, at a dose of $15 \mathrm{mg} / \mathrm{kg}$ body weight. At the end of the 4th week, $500 \mu 1$ blood was collected from the tail vein to evaluate glucose levels, and a fasting glucose of $\geq 11.1 \mathrm{mmol} / \mathrm{l}$ indicated that the T2DM model was successfully established. From the 5th to the 8th week, the diabetic rats were administered oxymatrine once per day (Fig. 1). The chemical structure of oxymatrine is shown in Fig. S1. The animals were randomly divided into 5 groups ( $\mathrm{n}=8$ per group) as follows: Control group, rats were fed a normal diet and injected with normal saline; model group, T2DM group; high-dose oxymatrine (CAS registry no. 2318-18-5; XiXisys Group Co., Ltd.); intervention group, T2DM rats were given oxymatrine at $20 \mathrm{mg} / \mathrm{kg}$ body weight by gavage; low-dose oxymatrine intervention group, T2DM rats were given oxymatrine at $10 \mathrm{mg} / \mathrm{kg}$ body weight by gavage; and metformin group, T2DM rats were given metformin at $25 \mathrm{mg} / \mathrm{kg}$ body weight. The levels of blood glucose and insulin sensitivity were assessed at the end of the 8th week. At the end of the experiments, all rats were sacrificed by acute blood loss by severing the femoral artery under anesthesia via intraperitoneal injection of 3\% pentobarbital sodium ( $40 \mathrm{mg} / \mathrm{kg}$ weight). After death was confirmed by lack of heartbeat and breathing, the liver tissues were collected and stored in liquid nitrogen for mRNA and protein analysis.

Measurement of fasting blood glucose levels. Blood samples of $500 \mu 1$ were obtained from the caudal vein of each rat using a needle (size 7) and the blood glucose levels were measured by a blood glucose meter (Sano).

Insulin sensitivity test in rats. A glucose-insulin tolerance test was used for the analysis of insulin sensitivity of rats. The rats were anesthetized by intraperitoneal injection of sodium pentobarbital at a dose of $50 \mathrm{mg} / \mathrm{kg}$ body weight following $8 \mathrm{~h}$ of fasting. The femoral vein and femoral artery were intubated following administration of glucose and insulin at $700 \mathrm{mg} / \mathrm{kg}$ and $0.175 \mathrm{U} / \mathrm{kg}$, respectively. Blood samples were collected from the femoral artery 3, 6, 9, 12 and 15-min after insulin injection, and the blood glucose levels were measured. The K-value was used for the analysis of sensitivity to insulin, which reflects the reduction rate of blood glucose between 3 and $15 \min (18)$.

Determination of body weight and liver index. The weights of the body and liver were measured using an electronic scale. The liver index was calculated as the ratio of liver weight to body weight.

Hematoxylin and eosin (HE) staining. The liver tissues were subjected to the following process: They were fixed in Carnoy's solution at $4^{\circ} \mathrm{C}$ for $20 \mathrm{~min}$, dehydrated in ethanol, transparentized in xylene, embedded in paraffin and cut into $5-\mu \mathrm{m}$ sections. The paraffin sections were then dewaxed and subjected to $\mathrm{HE}$ staining at $4^{\circ} \mathrm{C}$ for $10 \mathrm{~min}$. Then, morphological analysis was performed under a light microscope (magnification, x200; Olympus IX71, Olympus Corporation).

Cell culture and protocols. The HepG2 cell line was provided by China Center for Type Culture Collection. The cells were cultured in DMEM (Gibco; Thermo Fisher Scientific, Inc.) supplemented with 10\% FBS (Gibco; Thermo Fisher Scientific, Inc.), $100 \mathrm{U} / \mathrm{ml}$ penicillin and $100 \mu \mathrm{g} / \mathrm{ml}$ streptomycin, and cultured in a humidified incubator with $95 \%$ air and $5 \% \mathrm{CO}_{2}$ at $37^{\circ} \mathrm{C}$. The cells were subjected to different treatments after they had reached $70 \%$ confluence as follows: i) Control group, 


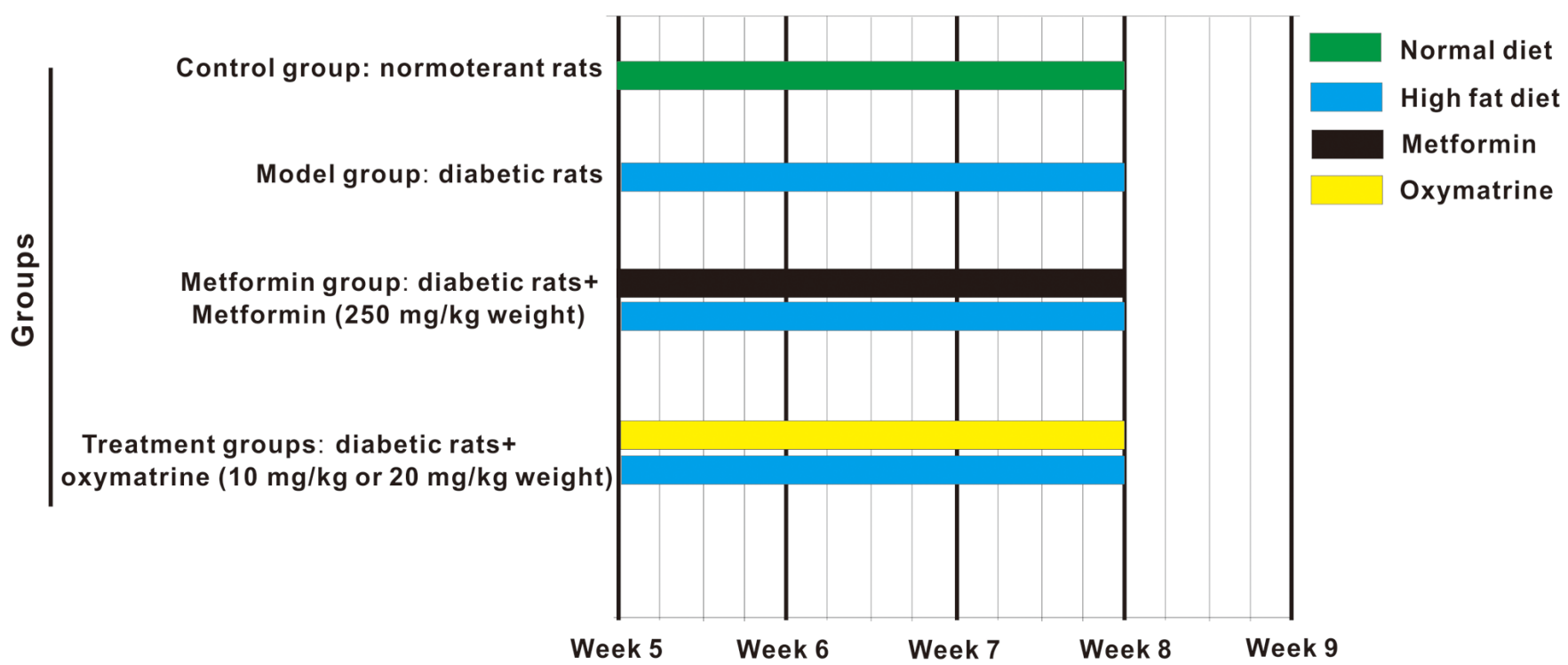

Figure 1. Schematic diagram of the time course of the experiment. Control group, normotolerant rats; model group, diabetic rats; treatment group, diabetic rats treated with oxymatrine (10 or $20 \mathrm{mg} / \mathrm{kg}$ body weight); and metformin group, diabetic rats treated with metformin $(250 \mathrm{mg} / \mathrm{kg}$ body weight).

cells were cultured in DMEM containing $5.5 \mathrm{mM}$ glucose for $24 \mathrm{~h}$; ii) high-glucose group, cells were cultured in DMEM containing $55 \mathrm{mM}$ glucose for $24 \mathrm{~h}$; iii) low-dose oxymatrine treatment group, cells were cultured in DMEM containing $55 \mathrm{mM}$ glucose and $0.1 \mu \mathrm{M}$ oxymatrine for $24 \mathrm{~h}$; iv) high-dose oxymatrine treatment group, cells were cultured in DMEM containing $55 \mathrm{mM}$ glucose and $1 \mu \mathrm{M}$ oxymatrine for $24 \mathrm{~h}$; v) metformin treatment group, cells were cultured in DMEM containing $55 \mathrm{mM}$ glucose and $0.1 \mu \mathrm{M}$ metformin for $24 \mathrm{~h}$; vi) oxymatrine and MK2206 combination treatment group, cells were cultured in DMEM containing $55 \mathrm{mM}$ glucose, $0.1 \mu \mathrm{M}$ oxymatrine and $3 \mu \mathrm{M}$ MK-2206 (AKT inhibitor; cat. no. SF2712, Beyotime Institute of Biotechnology) for $24 \mathrm{~h}$. Then, the cells were collected for mRNA and protein expression analysis, or glucose production and uptake assays.

Glucose production and uptake assays of HepG2 cells. For the glucose production assay, the HepG2 cells were rinsed with PBS to remove glucose and then incubated in glucose production assay medium (glucose- and phenol red-free DMEM) for $3 \mathrm{~h}$. Subsequently, the supernatant was collected for glucose concentration measurement by using a commercial colorimetric glucose assay kit (Sigma-Aldrich; Merck KGaA). The readings were then normalized to the total protein content determined from the whole-cell lysates.

The glucose uptake rate was measured using the methods established by Yoshioka et al (19) with slight modifications. Briefly, HepG2 cells were washed twice with PBS and then incubated with $200 \mu \mathrm{M} 2-\mathrm{NBDG}$ in glucose-free culture medium for $30 \mathrm{~min}$. Cells incubated with glucose-free medium without 2-NBDG served as a negative control. Finally, the cells were rinsed with PBS and fluorescence was determined using a microplate reader (Infinite M1000, Tecan Austria GmbH) with excitation at $488 \mathrm{~nm}$ and emission at $520 \mathrm{~nm}$.

RNA extraction and reverse transcription-quantitative $P C R(R T-q P C R)$ analysis. Total RNA from liver tissues was separated and extracted using an RNA extraction buffer (TRIzol ${ }^{\circledR}$, Takara Bio, Inc.), and the concentration and purity of RNA were determined by spectrophotometry. According to the operating instructions of the transcription kit (cat. no. DRR037A; Takara Bio, Inc.), 200 ng RNA was extracted from each sample for reverse transcription amplification. The 10- $\mu 1$ reverse transcription reaction system consisted of random primers $(0.5 \mu \mathrm{l})$, Oligo dT Primer $(0.5 \mu \mathrm{l})$, 5X PrimeScript buffer $(2 \mu \mathrm{l})$, RT Enzyme $(0.5 \mu \mathrm{l})$, RNase-Free $\mathrm{dH}_{2} \mathrm{O}(4.5 \mu \mathrm{l})$ and RNA $(2 \mu \mathrm{l})$. The reverse transcription reaction conditions were $37^{\circ} \mathrm{C}$ for $10 \mathrm{~min}$, followed by $85^{\circ} \mathrm{C}$ for $5 \mathrm{sec}$. Subsequently, the cDNA was mixed with SYBR premix reagent (Takara Bio, Inc.) and primers to create a $25-\mu 1$ reaction system $\left(10 \mu \mathrm{l}\right.$ SYBR Premix Ex Taq ${ }^{\mathrm{TM}}, 0.5 \mu 1$ forward primer, $0.5 \mu \mathrm{l}$ reverse primer, $0.5 \mu \mathrm{l} 50 \mathrm{X}$ ROX Reference Dye, $4 \mu \mathrm{l}$ RNA and $4.5 \mu \mathrm{l}$ RNase-Free $\mathrm{dH}_{2} \mathrm{O}$ ). The thermocycling conditions were as follows: Pre-denaturation $\left(95^{\circ} \mathrm{C}, 30 \mathrm{sec}\right)$, denaturation $\left(95^{\circ} \mathrm{C}, 30 \mathrm{sec}\right)$, annealing $\left(60^{\circ} \mathrm{C}, 30 \mathrm{sec}\right)$ and elongation $\left(70^{\circ} \mathrm{C}, 31 \mathrm{sec}\right)$, for a total of 35 cycles. The sequences of the PCR primers used were as follows: PEPCK forward, 5'-GGATGTGGCCAGGATCGAAA-3' and reverse, 5'-ATA CATGGTGCGGCCTTTCA-3'; G6Pase forward, 5'-GAT TCCGGTGCTTGAATGTCG-3' and reverse, 5'-GCATTG TAGATGCCCCGGAT-3'; and $\beta$-actin forward, 5'-CCCATC TATGAGGGTTACGC-3' and reverse, 5'-TTTAATGTCACG CACGATTTC-3'. The mRNA levels of PEPCK and G6Pase in the liver were analyzed using an ABI 7300 amplifier (Thermo Fisher Scientific, Inc.). The $2^{-\Delta \Delta \mathrm{Cq}}$ method was used for data analysis and results were normalized to $\beta$-actin (20).

Western blot analysis. Tissues or cells were treated with lysis buffer containing PMSF (1\% mmol/1; cat. no. ST505, Beyotime Institute of Biotechnology) and Phosphatase inhibitor cocktail A (cat. no. P1081, Beyotime Institute of Biotechnology), and total protein was obtained. A BCA protein assay kit (cat. no. P0001, Beyotime Institute of Biotechnology) was used for protein concentration determination. Then, the proteins 
were denatured at $99^{\circ} \mathrm{C}$ for $5 \mathrm{~min}$. Following denaturation, $40 \mu \mathrm{g}$ protein from each sample was loaded per lane, subjected to $10 \%$ SDS-PAGE and transferred to a PVDF membrane, which was then blocked using $20 \%$ skimmed milk powder in TBS-T. Subsequently, the membrane was rinsed with water and incubated with primary antibodies (dilution 1:1,000) against PEPCK (cat. no. sc-271029, Santa Cruz Biotechnology, Inc.), G6Pase (cat. no. sc-25840, Santa Cruz Biotechnology, Inc.), AKT (cat. no. sc-81434, Santa Cruz Biotechnology, Inc.), pAKT (cat. no. sc-514032, Santa Cruz Biotechnology, Inc.) and $\beta$-actin (cat. no. AF5001, Beyotime Institute of Biotechnology) overnight at $4^{\circ} \mathrm{C}$. Then, the membrane was rinsed with Tris-EDTA buffer and water, followed by a 2 -h incubation at $25^{\circ} \mathrm{C}$ with horseradish peroxidase-conjugated secondary antibodies (cat. no. A0208, goat anti-rabbit IgG; cat. no. A0216, goat anti-mouse IgG; dilution 1:2,000, Beyotime Institute of Biotechnology). The signals were visualized using an enhanced chemiluminescence kit (GE Healthcare). ImageJ version 1.43 (National Institutes of Health) was used for optical density analysis. In order to ensure the accuracy of sample addition, $\beta$-actin was used as the loading control.

Statistical analysis. SPSS software (version 17; SPSS, Inc.) was used for statistical analysis. The data are expressed as the mean \pm standard deviation. A one-way ANOVA followed by a Tukey's post hoc test was used for comparison between multiple groups. All experiments were repeated three times. $\mathrm{P}<0.05$ was considered to indicate a statistically significant difference.

\section{Results}

Effects of oxymatrine on fasting blood glucose in T2DM rats. After inducing T2DM in SD rats with a high-fat diet and intraperitoneal injection of streptozotocin, the fasting blood glucose levels of the rats was measured. As shown in Fig. 2, compared with the control group $(6.1 \mathrm{mmol} / \mathrm{l})$, the fasting blood glucose levels of T2DM rats were increased significantly $(16.5 \mathrm{mmol} / \mathrm{l})$, indicating that the T2DM model was successfully established. Then, the T2DM rats were administered oxymatrine and it was observed that oxymatrine could reduce the fasting blood glucose levels in a dose-dependent manner (Fig. 2). This suggested that oxymatrine may be effective for lowering blood glucose concentration.

Effects of oxymatrine on insulin sensitivity in T2DM rats. Considering that the levels of blood glucose are closely associated with insulin activity, the next experiment was designed to observe the role of oxymatrine in the insulin sensitivity of T2DM rats. As shown in Fig. 3, compared with the control, the insulin sensitivity of T2DM rats was significantly decreased, while oxymatrine improved the insulin sensitivity of T2DM rats in a dose-dependent manner. These results indicated that oxymatrine may increase the sensitivity of insulin in T2DM rats.

Effects of oxymatrine on the expression of AKT, PEPCK and G6Pase in the liver of T2DM rats. As the increase in hepatic gluconeogenesis caused by insulin resistance is an important pathological characteristic of T2DM, the effect of oxymatrine on the expression of PEPCK and G6Pase in liver tissues was

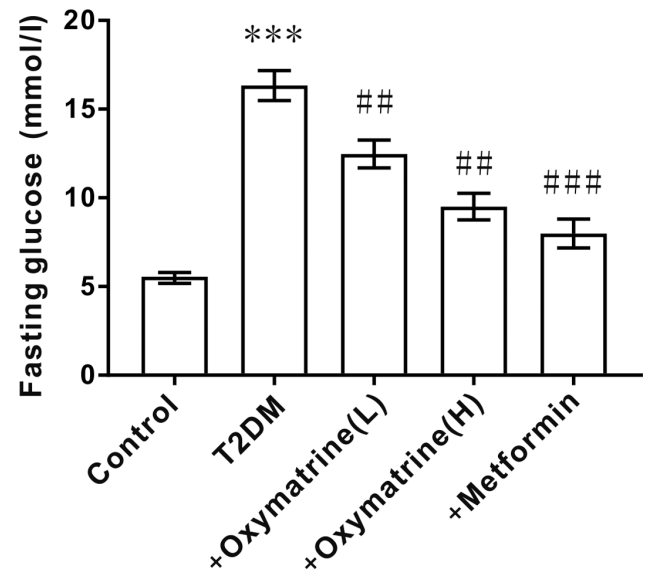

Figure 2. Effect of oxymatrine on fasting blood glucose in T2DM rats. The differences in the values between groups were analyzed using a one-way ANOVA. All experiments were repeated 3 times and all data are presented as the mean \pm standard deviation. ${ }^{* * *} \mathrm{P}<0.001 \mathrm{vs}$. control; ${ }^{\# \#} \mathrm{P}<0.01,{ }^{\# \# \#} \mathrm{P}<0.001$ vs. T2DM. T2DM, type 2 diabetes mellitus; +oxymatrine (L), type 2 diabetic rats treated with $10 \mathrm{mg} / \mathrm{kg}$ body weight oxymatrine; +oxymatrine $(\mathrm{H})$, type 2 diabetic rats treated $20 \mathrm{mg} / \mathrm{kg}$ body weight oxymatrine; +Metformin, type 2 diabetic rats treated with $250 \mathrm{mg} / \mathrm{kg}$ body weight metformin.

examined. As shown in Fig. 4A-E, compared with the control group, the mRNA and protein levels of PEPCK and G6Pase in the livers of T2DM rats were significantly increased, and oxymatrine dose-dependently inhibited the expression of PEPCK and G6Pase. Due to the important role of AKT in T2DM, the effect of oxymatrine on the expression of AKT in the rat liver was examined. As shown in Fig. $4 \mathrm{C}$ and F, compared with the control group, the phosphorylation levels of AKT in the livers of T2DM rats was significantly decreased, and oxymatrine dose-dependently increased the phosphorylation levels of AKT in the livers of T2DM rats. These results indicated that the protective function of oxymatrine in T2DM rats may be associated with the inhibition of gluconeogenesis and the regulation of AKT phosphorylation.

Effects of oxymatrine on liver index and liver tissue morphology in T2DM rats. The next experiment was performed to observe the effect of oxymatrine on the body weight, liver weight and liver index of T2DM rats. The results revealed a notable decrease in body weight, and an increase in liver weight and liver index of diabetic rats, whereas oxymatrine dose-dependently reversed these phenomena (Fig. 5A-C). HE staining revealed that the liver tissues of diabetic rats exhibited obvious morphological changes, such as karyopyknosis and fibrosis, whereas oxymatrine dose-dependently prevented the morphological changes to liver tissues (Fig. 5D). These results indicated that oxymatrine exerts hepatoprotective effects.

Effects of oxymatrine on glucose production and uptake in Hep $\mathrm{G} 2$ cells. To observe whether oxymatrine can inhibit the gluconeogenesis of liver cells under diabetic conditions, HepG2 cells were treated with different concentrations of glucose and oxymatrine. As shown in Fig. 6A, the HepG2 cells treated with high glucose $(55 \mathrm{mM})$ exhibited higher glucose production activity when compared with the control cells, and oxymatrine significantly reduced glucose production in a dose-dependent manner (Fig. 6A). 

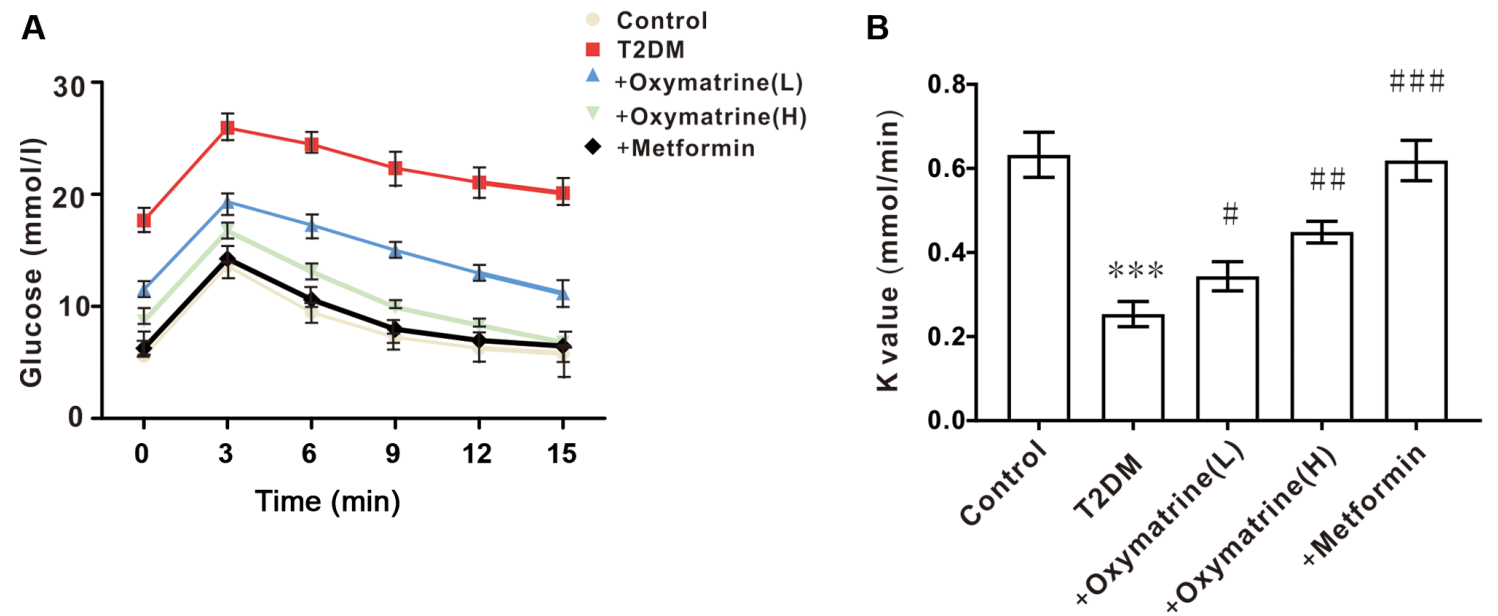

Figure 3. Effect of oxymatrine on insulin sensitivity in T2DM rats. (A) Blood glucose levels and (B) K value in the treated rats. Differences between groups were compared using a one-way ANOVA. All experiments were repeated 3 times and all data are presented as the mean \pm standard deviation. ${ }^{* * *} \mathrm{P}<0.001$ vs. control; ${ }^{\#} \mathrm{P}<0.05,{ }^{\# \#} \mathrm{P}<0.01,{ }^{\# \#} \mathrm{P}<0.001$ vs. T2DM. T2DM, type 2 diabetes mellitus; +oxymatrine (L), type 2 diabetic rats treated with 10 mg/kg body weight oxymatrine; +oxymatrine $(\mathrm{H})$ type 2 diabetic rats treated $20 \mathrm{mg} / \mathrm{kg}$ body weight oxymatrine; +Metformin, type 2 diabetic rats treated with $250 \mathrm{mg} / \mathrm{kg}$ body weight metformin

A

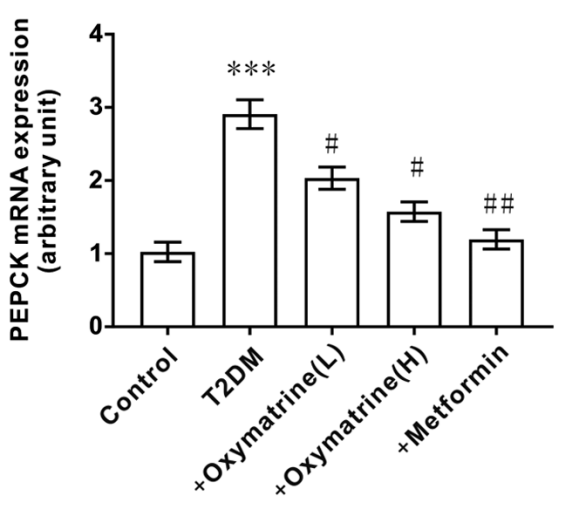

D

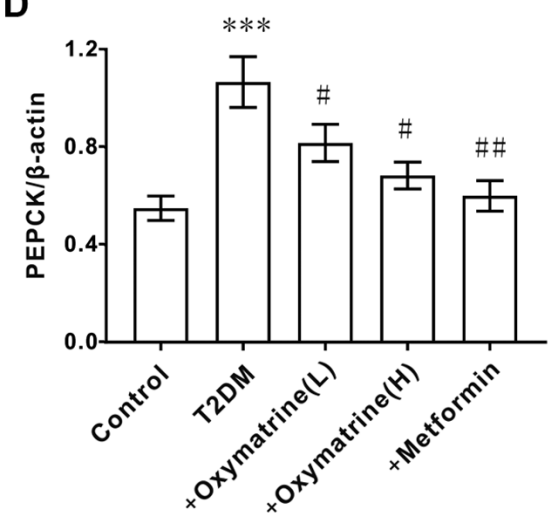

B

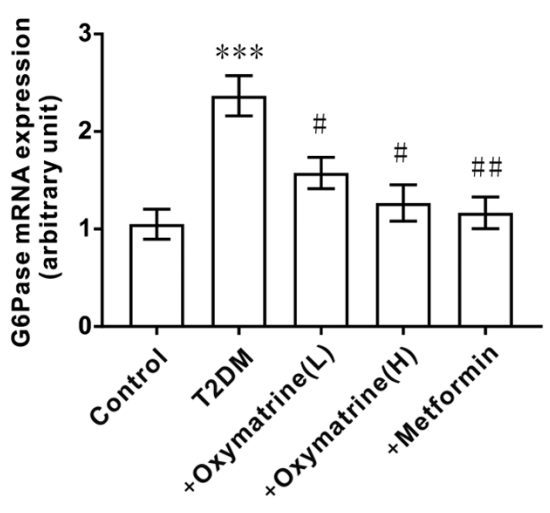

E

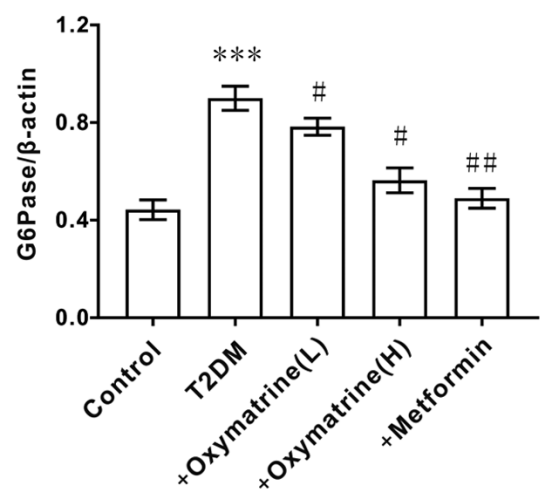

C

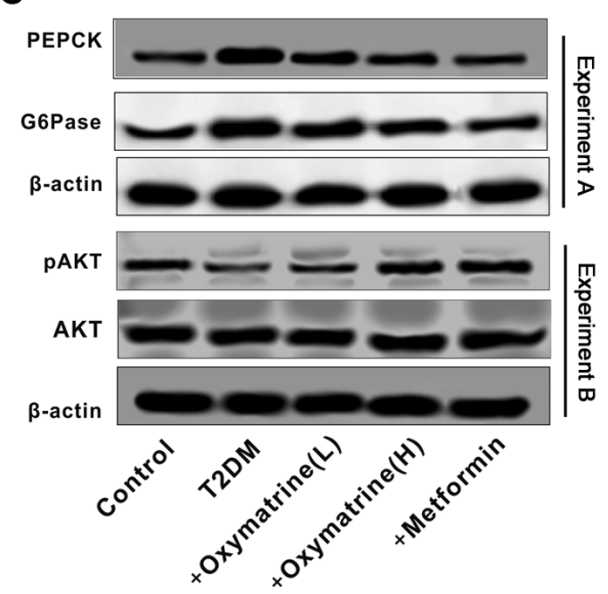

F

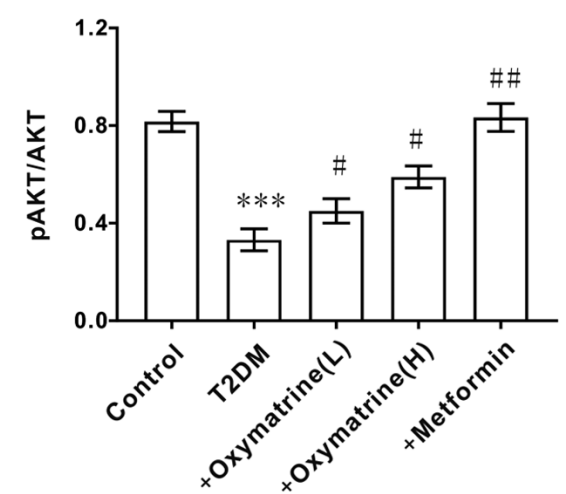

Figure 4. Effect of oxymatrine on the expression of AKT, PEPCK and G6Pase in the liver of type 2 diabetic rats. (A) mRNA expression levels of PEPCK and (B) G6Pase. (C) Protein expression levels of PEPCK, G6Pase, AKT and pAKT. (D) Ratio of PEPCK to $\beta$-actin. (E) Ratio of G6Pase to $\beta$-actin. (F) Ratio of pAKT to $\beta$-actin. Differences between groups were compared using a one-way ANOVA. All experiments were repeated 3 times and all data are presented as the mean \pm standard deviation. ${ }^{* * *} \mathrm{P}<0.05$ vs. control; ${ }^{\#} \mathrm{P}<0.05,{ }^{\# \#} \mathrm{P}<0.01$ vs. T2DM. T2DM, type 2 diabetes mellitus; +oxymatrine (L) type 2 diabetic rats treated with $10 \mathrm{mg} / \mathrm{kg}$ body weight oxymatrine; +oxymatrine $(\mathrm{H})$ type 2 diabetic rats treated $20 \mathrm{mg} / \mathrm{kg}$ body weight oxymatrine; +Metformin, type 2 diabetic rats treated with $250 \mathrm{mg} / \mathrm{kg}$ body weight metformin; p, phospho; PEPCK, phosphoenolpyruvate carboxykinase; glucose-6-phosphatase.

The next experiment was designed to observe the effect of oxymatrine on the glucose uptake ability of HepG2 cells.
As shown in Fig. 6B, the HepG2 cells treated with high glucose $(55 \mathrm{mM})$ exhibited weaker glucose uptake ability, 
A

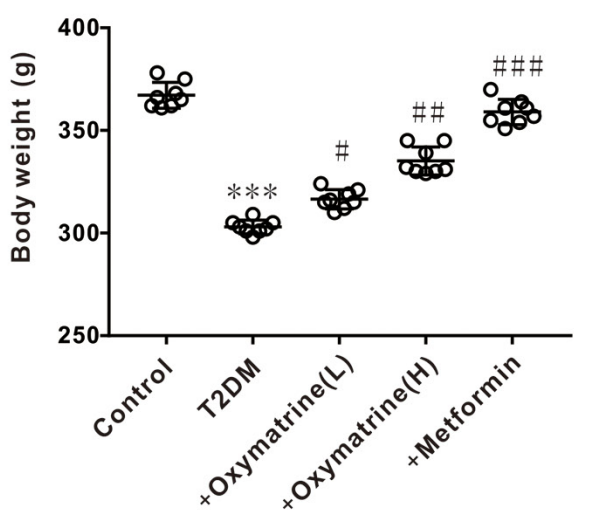

B

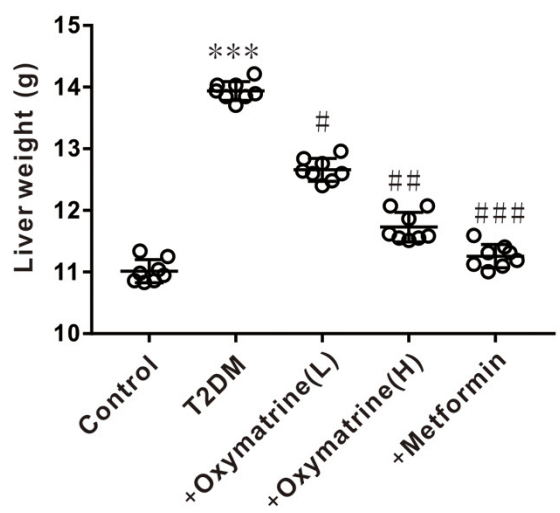

C

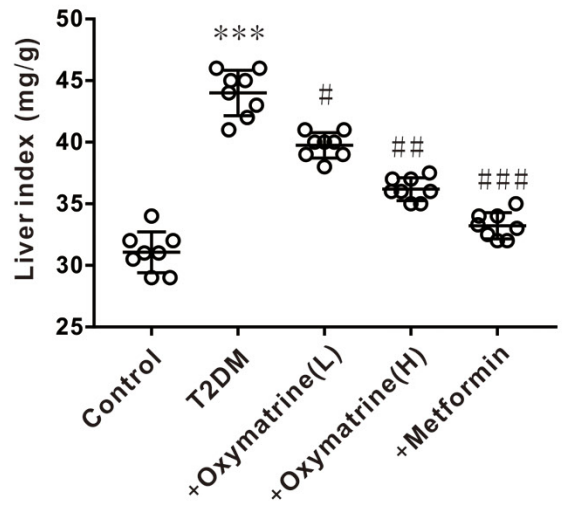

D

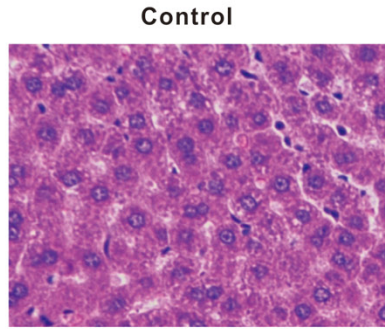

+Oxymatrine $(\mathrm{H})$

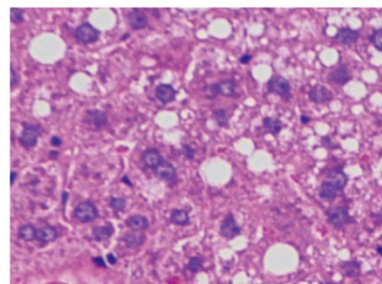

T2DM

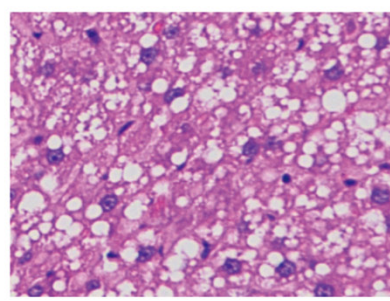

+Metformin

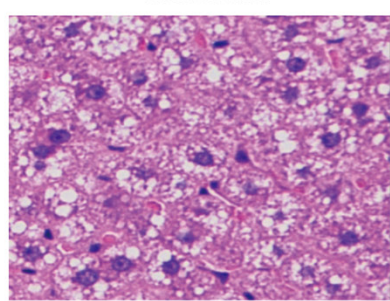

+Oxymatrine $(\mathrm{L})$

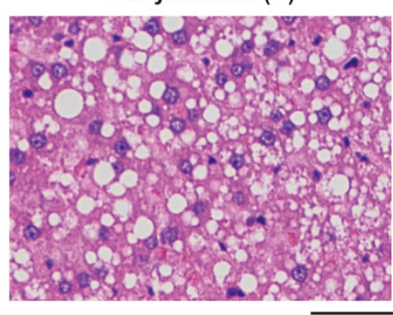

$50 \mu \mathrm{m}$

Figure 5. Effects of oxymatrine on liver index and liver tissue morphology of the T2DM rats. (A) Body weight, (B) liver weight and (C) liver index of the treated rats. (D) Representative images of HE staining of the liver. Differences between groups were compared using a one-way ANOVA. All experiments were repeated 3 times and all data are presented as the mean \pm standard deviation. ${ }^{* * *} \mathrm{P}<0.001$ vs. control; ${ }^{\#} \mathrm{P}<0.05,{ }^{\# \#} \mathrm{P}<0.01,{ }^{\# \# \#} \mathrm{P}<0.001$ vs. T2DM. T2DM. T2DM, type 2 diabetes mellitus; +oxymatrine (L), type 2 diabetic rats treated with $10 \mathrm{mg} / \mathrm{kg}$ body weight oxymatrine; +oxymatrine (H) type 2 diabetic rats treated $20 \mathrm{mg} / \mathrm{kg}$ body weight oxymatrine; +Metformin, type 2 diabetic rats treated with $250 \mathrm{mg} / \mathrm{kg}$ body weight metformin; HE, hematoxylin and eosin.

A

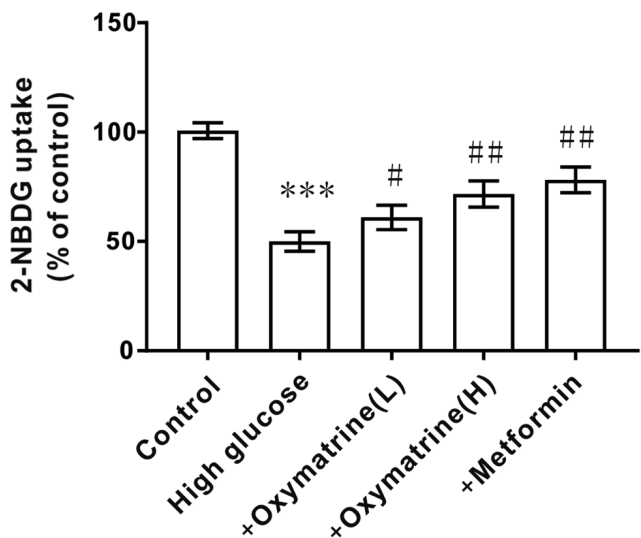

B

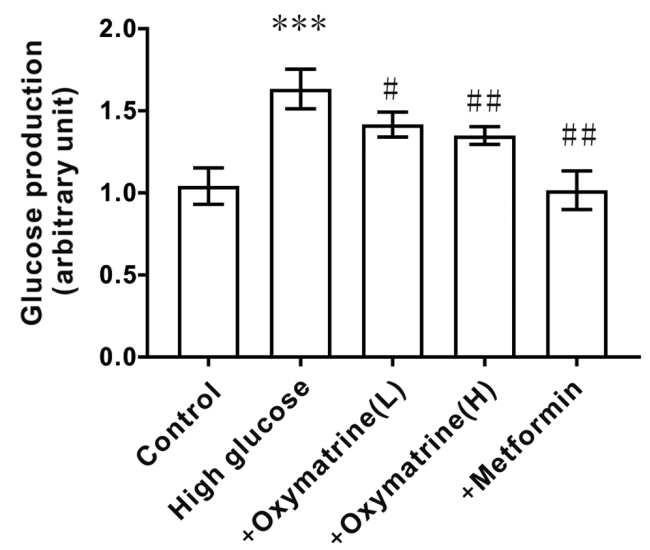

Figure 6. Effect of oxymatrine on glucose production and uptake in HepG2 cells. (A) glucose production assay, and (B) 2-NBDG uptake assay in the treated cells. Differences between groups were compared using a one-way ANOVA. All experiments were repeated 3 times and all data are presented as the mean \pm standard deviation. ${ }^{* * *} \mathrm{P}<0.001$ vs. control; ${ }^{*} \mathrm{P}<0.05,{ }^{\# \#} \mathrm{P}<0.01$ vs. High Glucose. +oxymatrine (L) cells treated with $0.1 \mu \mathrm{M}$ oxymatrine; +oxymatrine (H) cells treated with $1 \mu \mathrm{M}$ oxymatrine; +Metformin, cells treated with $0.1 \mu \mathrm{M}$ metformin.

and oxymatrine improved the glucose uptake ability of high-glucose-treated cells in a dose-dependent manner.
These data suggested that oxymatrine may promote liver cell gluconeogenesis. 
A
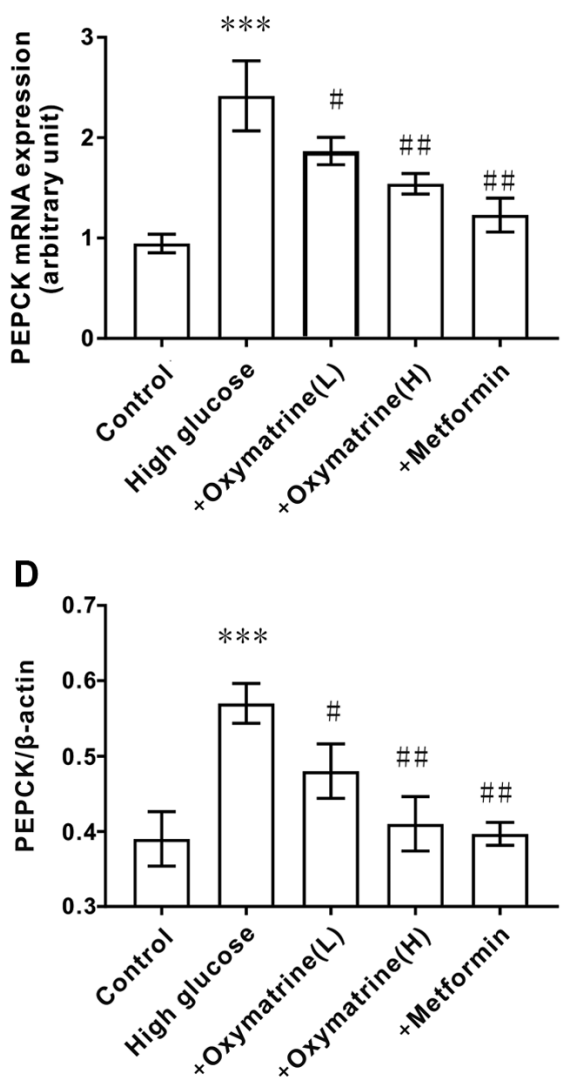

B

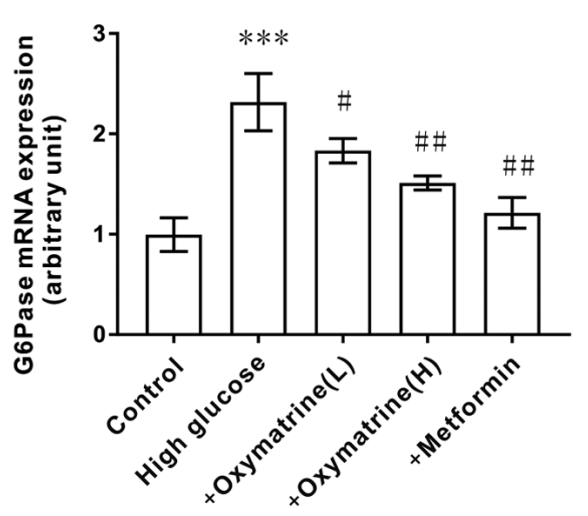

E

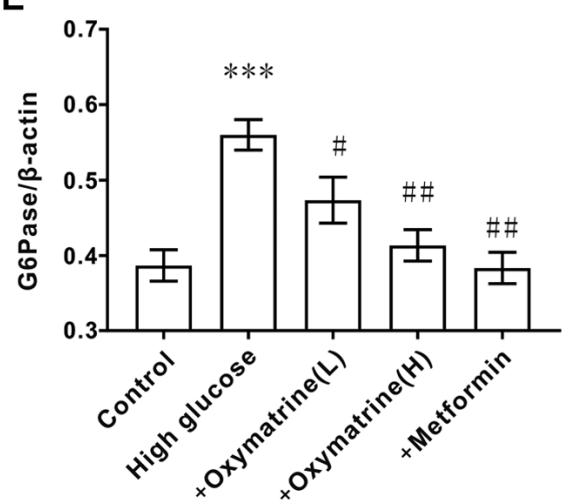

C

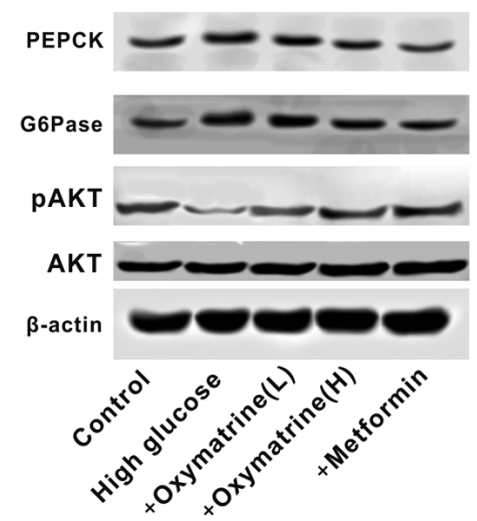

F

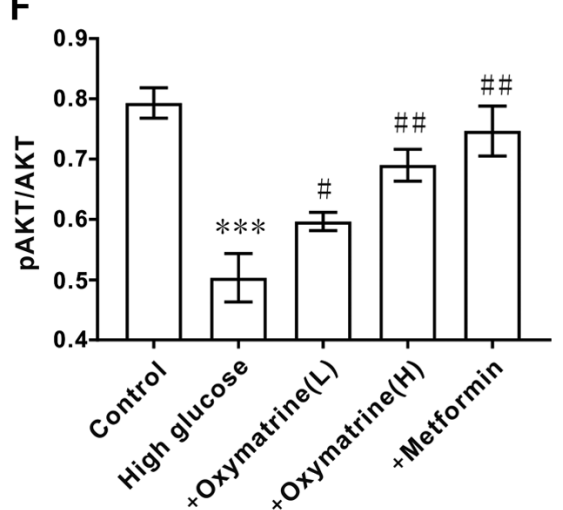

Figure 7. Effect of oxymatrine on the expression of AKT, PEPCK and G6Pase in HepG2 cells. (A) mRNA expression levels of PEPCK and (B) G6Pase in the treated cells. (C) Protein expression of PEPCK, G6Pase, AKT and pAKT. (D) Ratio of PEPCK, (E) G6Pase and (F) pAKT to $\beta$-actin. Differences between groups were compared using a one-way ANOVA. All experiments were repeated 3 times and all data are presented as the mean \pm standard deviation. ${ }^{* * *} \mathrm{P}<0.001$ vs. control; ${ }^{\#} \mathrm{P}<0.05,{ }^{\# \#} \mathrm{P}<0.01$ vs. High Glucose. +oxymatrine $(\mathrm{L})$ cells treated with $0.1 \mu \mathrm{M}$ oxymatrine; +oxymatrine $(\mathrm{H})$ cells treated with $1 \mu \mathrm{M}$ oxymatrine; +Metformin, cells treated with $0.1 \mu \mathrm{M}$ metformin; PEPCK, phosphoenolpyruvate carboxykinase; glucose-6-phosphatase; p, phospho.

Effects of oxymatrine on the expression of PEPCK and G6Pase and the phosphorylation of AKT in HepG2 cells. The effect of oxymatrine on the expression of gluconeogenesis-related genes in liver cells was next assessed. As shown in Fig. 7, when compared with the control cells, HepG2 cells treated with high glucose $(55 \mathrm{mM})$ exhibited significantly increased expression of PEPCK and G6Pase at both the mRNA and protein levels, but decreased phosphorylation of AKT. In accordance with the animal experimental results, oxymatrine significantly inhibited the expression of PEPCK and G6Pase at both the mRNA and protein level in HepG2 cells, and increased the phosphorylation level of AKT. These data indicated that the effect of oxymatrine on gluconeogenesis was associated with the regulation of PEPCK and G6Pase expression, as well as AKT phosphorylation.

$M K-2206$ reverses the effect of oxymatrine on the expression of PEPCK and G6Pase, and the phosphorylation of AKT in HepG2 cells. To further observe whether the regulatory effect of oxymatrine on the expression of PEPCK and G6Pase was associated with the regulation of AKT phosphorylation, HepG2 cells were treated with oxymatrine in combination with the AKT inhibitor, MK-2206. As shown in Fig. 8A and B, oxymatrine significantly increased AKT phosphorylation in HepG2 cells subjected to high glucose treatment, and this effect was reversed when the cells were treated with MK-2206.
The MK-2206 and oxymatrine combination treatment significantly increased the expression of PEPCK and G6Pase when compared with oxymatrine alone (Fig. 8C-G). These data suggested that the effect of oxymatrine on the expression of PEPCK and G6Pase was associated with the regulation of AKT phosphorylation.

\section{Discussion}

The present study demonstrated that the rats fed with a high-fat diet and injected with streptozotocin, or HepG2 cells treated with high concentrations of glucose, exhibited reduced phosphorylation of AKT and high expression of PEPCK and G6Pase, whereas oxymatrine dose-dependently reversed these phenomena. It was also observed that the AKT inhibitor significantly reversed the effect of oxymatrine on AKT phosphorylation, and PEPCK and G6Pase expression in HepG2 cells. This indicated that the inhibitory effect of oxymatrine against gluconeogenesis was associated with the regulation of the expression of PEPCK and G6Pase, which was at least partly associated with the regulation of AKT phosphorylation in the liver (Fig. S2).

AKT has an important function in insulin signaling, and its dysfunction causes insulin resistance, which is a characteristic of T2DM (6). AKT participates in numerous biological functions in mammalians, such as cell cycle regulation, cell 

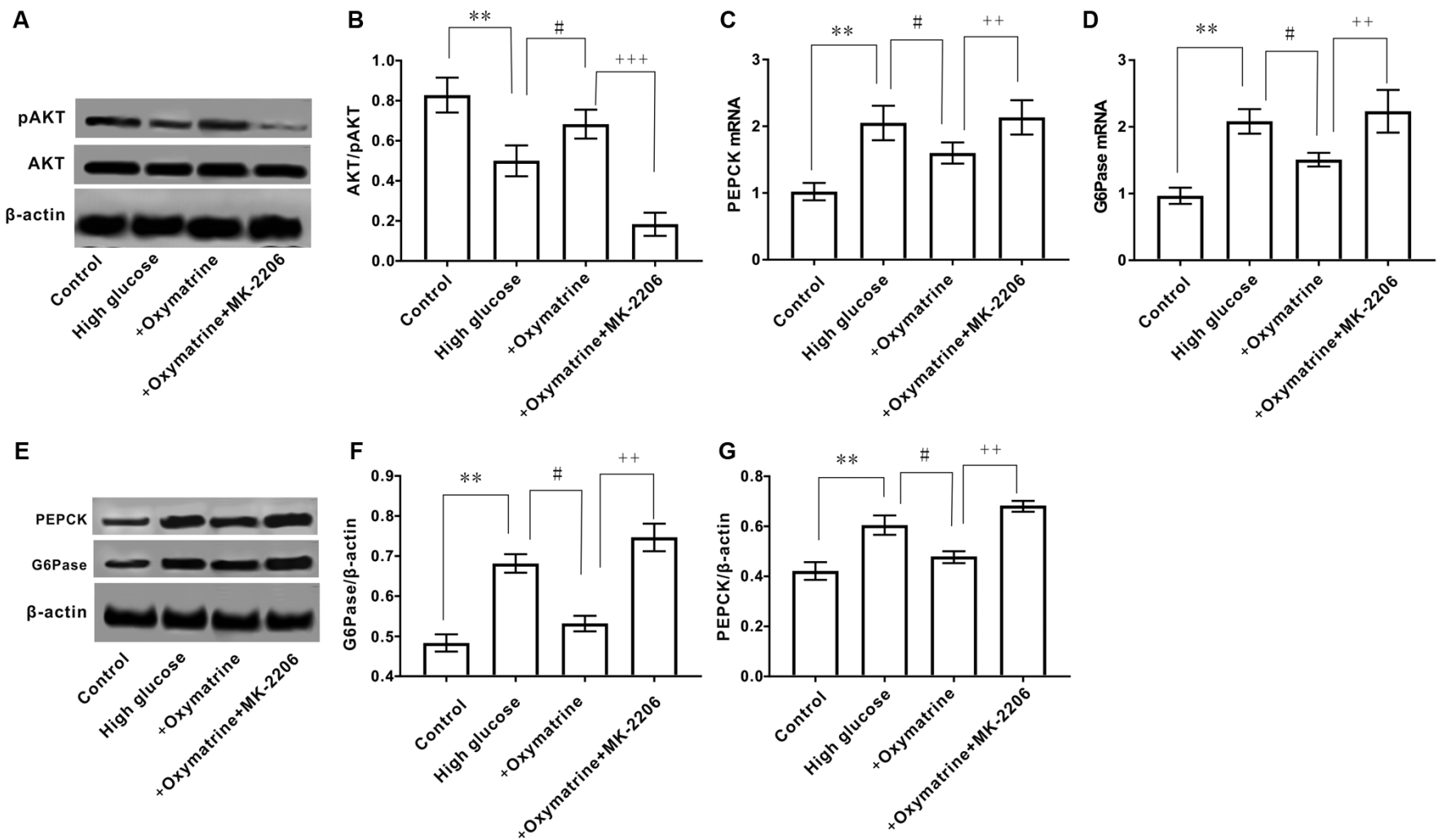

Figure 8. Effect of inhibition of AKT on the expression of AKT, PEPCK and G6Pase in HepG2 cells. (A) Protein expression of AKT and pAKT. (B) Ratio of pAKT to AKT. mRNA expression levels of (C) PEPCK and (D) G6Pase. (E) Protein expression of PEPCK and G6Pase. Ratio of (F) G6Pase to $\beta$-actin and (G) PEPCK to $\beta$-actin. Differences between groups were compared using a one-way ANOVA. All experiments were repeated 3 times and all data are presented as the mean \pm standard deviation. ${ }^{* *} \mathrm{P}<0.01$ vs. control; ${ }^{~} \mathrm{P}<0.05$ vs. High Glucose; ${ }^{++} \mathrm{P}<0.01,{ }^{++} \mathrm{P}<0.001$ vs. +oxymatrine. PEPCK, phosphoenolpyruvate carboxykinase; G6Pase, glucose-6-phosphatase.

survival, glucose metabolism, protein synthesis and NO production, by phosphorylating its downstream target genes (21). However, activation of AKT itself is also regulated by several factors, such as insulin-like growth factor and transforming growth factor (21). The inactivation of AKT is associated with the dephosphorylation of AKT phosphorylase, PI3K, and is mediated by PTEN (21). The present and previous studies found that the levels of AKT phosphorylation in the liver of T2DM rats decreased significantly, indicating that abnormal AKT expression is an important factor implicated in the occurrence and development of T2DM (22-24). Therefore, the AKT signaling pathway may serve an important role in blood glucose homeostasis, and insulin/AKT signaling may represent a potential target for T2DM therapy.

The liver is the primary site of gluconeogenesis, which can convert non-sugar substances, such as glycerin, lactic acid and glycogenic amino acids, into glucose. The process of gluconeogenesis is regulated by a number of factors, such as the regulation of glycogenic substrates, key enzymes and transcription factors, amongst which, the regulation of gluconeogenesis rate-limiting enzymes is particularly important (4). Pyruvate, as the key substrate of gluconeogenesis, is converted to oxaloacetic acid by pyruvate carboxylase and malate dehydrogenase, and then to phosphoenolpyruvate by PEPCK; phosphoenolpyruvate undergoes multiple chemical reactions and is converted to fructose-1,6-diphosphate, which is further converted to fructose-6-phosphate; fructose-6-phosphate is then converted into glucose-6-phosphate and transported to the endoplasmic reticulum, where it is converted into glucose under the action of G6Pase (4). PEPCK and G6Pase are both key and rate-limiting enzymes in gluconeogenesis, and this has been confirmed by experiments in which PEPCK knockout mice lost the ability to synthesize glucose using lactic acid, which adversely affected survival, and G6Pase knockout mice exhibited symptoms such as hyperlipidemia and lactic acid accumulation $(25,26)$. The regulation of PEPCK expression is a complex biological process. PEPCK can be acetylated by histone acetylase P300 and then labeled by ubiquitin and subjected to proteasome degradation, while Sirt2 can deacetylate PEPCK and increase its expression (27). In addition, the expression of PEPCK and G6Pase may also be regulated by several transcription factors, such as cAMP response element-binding protein (CREB), FoxO1 and CCAAT enhancer-binding protein $\alpha / \beta$ (28-30). Inhibition of the transcription activity of CREB significantly decreased the expression of PEPCK and G6Pase, which was accompanied by reduced glucose production (31). Moreover, it was reported that the expression of CREB and FoxO1 may be regulated by the AKT signaling pathway and the expression of PEPCK and G6Pase may be associated with AKT phosphorylation deficiency $(32,33)$. Consistently, the present study demonstrated that AKT phosphorylation in the liver of T2DM rats was significantly decreased, while the expression of PEPCK and G6Pase was significantly increased, which was accompanied by an increase in blood glucose concentration. The results demonstrated that when AKT phosphorylation was 
dysregulated, the key enzymes of gluconeogenesis, PEPCK and G6Pase, were upregulated, and gluconeogenesis was significantly enhanced in the livers of T2DM rats.

In view of the important role of insulin resistance and gluconeogenesis in the occurrence and development of T2DM, inhibiting insulin resistance and gluconeogenesis may prove to be valuable methods for prevention and therapy of T2DM. However, some of the drugs currently used for treatment of T2DM are not specific, for example, rosiglitazone and metformin, and drug resistance usually develops after long-term use (34). Therefore, novel drugs for T2DM are urgently required. China has a long history of research and application of Chinese herbal medicines $(12,35)$. Screening out the effective components related to insulin resistance and gluconeogenesis in herbal medicines that exhibit beneficial effects against insulin, may be an effective method for identifying novel drugs for T2DM therapy (12). It was previously reported that oxymatrine can protect obese rats from injury by reducing the triglyceride and total cholesterol levels, and increasing serum insulin content (35). In addition, oxymatrine can increase the synthesis of glycogen (including liver and muscle glycogen) and the expression levels of glucose transporter 4 (35). Our previous study found that oxymatrine protects diabetic rats from insulin resistance through the regulation of phosphorylation of AKT (18). These results suggest that the function of oxymatrine in protecting diabetic rats from hyperglycemia may be associated with insulin resistance and gluconeogenesis. Of note, a clinical trial performed by $\mathrm{Yu}$ et al (36) demonstrated that the administration of oxymatrine in combination with metformin was beneficial for diabetic patients by inhibiting the symptoms of insulin resistance. The present study demonstrated that oxymatrine exerted protective effects in T2DM rats by improving insulin sensitivity and reducing the blood glucose levels. The underlying mechanism involved the regulation of the expression of PEPCK, G6Pase and AKT in the liver, which suggests that oxymatrine may be a novel candidate drug for T2DM therapy. However, one limitation of the present study was the use of only male rats for the experiments, as the possible differences in oxymatrine action according to sex could not be evaluated. Therefore, further studies are required to address these issues.

In conclusion, to the best of our knowledge, the present study was the first to demonstrate that the effect of oxymatrine against gluconeogenesis was associated with the regulation of PEPCK and G6Pase expression in the liver, and that its effects were at least partly mediated via regulation of AKT phosphorylation.

\section{Acknowledgements}

Not applicable.

\section{Funding}

This work was supported by HuNan Provincial Science \& Technology Department (grant nos. 2020JJ5384 and 2020JJ4442); HuNan Health Commission (grant no. C20200350), and ChangSha Science \& Technology Bureau (grant no. kq1801126).

\section{Availability of data and materials}

The dataset used and/or analyzed during the present study are available from the corresponding author on reasonable request.

\section{Authors' contributions}

YXZ, HQH, MLZ, TML and LM performed the experiments. LCD, LM and MDSAS contributed to the data analysis and manuscript drafting. GLS and ZBY contributed to the design of the experiments and drafting of the manuscript. All authors have read and approved the manuscript. YXZ and ZBY confirmed the authenticity of all the raw data.

\section{Ethics approval and consent to participate}

The study was performed in accordance with the National Research Council (US) Committee guidelines for the care and use of experimental animals and was approved by the Veterinary Animal Care and use Committee of Hunan Normal University (approval no. 2019111).

\section{Patient consent for publication}

Not applicable.

\section{Competing interests}

The authors declare that they have no competing interests.

\section{References}

1. American Diabetes Association: Diagnosis and classification of diabetes mellitus. Diabetes Care 37 (Suppl 1): S81-S90, 2014.

2. Ma RCW: Epidemiology of diabetes and diabetic complications in China. Diabetologia 61: 1249-1260, 2018.

3. Wu Y, Ding Y, Tanaka Y and Zhang W: Risk factors contributing to type 2 diabetes and recent advances in the treatment and prevention. Int J Med Sci 11: 1185-1200, 2014.

4. Rines AK, Sharabi K, Tavares CD and Puigserver P: Targeting hepatic glucose metabolism in the treatment of type 2 diabetes. Nat Rev Drug Discov 15: 786-804, 2016.

5. Rui L: Energy metabolism in the liver. Compr Physiol 4: 177-197, 2014.

6. De Meyts P: The insulin receptor and its signal transduction network. [Updated 2016 Apr 27]. In. Feingold KR, Anawalt B, Boyce A, Chrousos G, de Herder WW, Dhatariya K, Dungan K, Grossman A, Hershman JM, Hofland J, et al (eds). Endotext (Internet). South Dartmouth (MA), MDText.com, Inc., 2000. https://www.ncbi.nlm.nih.gov/books/NBK378978. Accessed April 27, 2016

7. Muoio DM and Newgard CB: Mechanisms of disease: Molecular and metabolic mechanisms of insulin resistance and beta-cell failure in type 2 diabetes. Nat Rev Mol Cell Biol 9: 193-205, 2008.

8. Fan H, Li L, Zhang X, Liu Y, Yang C, Yang Y and Yin J: Oxymatrine downregulates TLR4, TLR2, MyD88 and NF-kappa B and protects rat brains against focal ischemia. Mediators Inflamm 2009: 704706, 2009.

9. Liu Y, Xu Y, Ji W, Li X, Sun B, Gao Q and Su C: Anti-tumor activities of matrine and oxymatrine: Literature review. Tumour Biol 35: 5111-5119, 2014.

10. Chen XS, Wang GJ, Cai X, Yu HY and Hu YP: Inhibition of hepatitis B virus by oxymatrine in vivo. World J Gastroenterol 7: 49-52, 2001.

11. Wu XL, Zeng WZ, Jiang MD, Qin JP and Xu H: Effect of oxymatrine on the TGF beta-Smad signaling pathway in rats with CCl4-induced hepatic fibrosis. World J Gastroenterol 14: 2100-2105, 2008 
12. Chan SM and Ye JM: Strategies for the discovery and development of anti-diabetic drugs from the natural products of traditional medicines. J Pharm Pharm Sci 16: 207-216, 2013.

13. Wang L, Li X, Zhang Y,Huang Y,Zhang Y and Ma Q: Oxymatrine ameliorates diabetes-induced aortic endothelial dysfunction via the regulation of eNOS and NOX4. J Cell Biochem, Nov 19, 2018 (Epub ahead of print). doi: 10.1002/jcb.28006.

14. Wang SB and Jia JP: Oxymatrine attenuates diabetes-associated cognitive deficits in rats. Acta Pharmacol Sin 35: 331-338, 2014.

15. Lu ML, Xiang XH and Xia SH: Potential signaling pathways involved in the clinical application of oxymatrine. Phytother Res 30: 1104-1112, 2016.

16. National Research Council (US) Committee for the update of the Guide for the Care and Use of Laboratory Animals: Guide for the Care and Use of Laboratory Animals. 8th edition. National Academicals Press, Washington, DC, 2011.

17. Ma C, Yu H, Xiao Y and Wang H: Momordica charantia extracts ameliorate insulin resistance by regulating the expression of SOCS-3 and JNK in type 2 diabetes mellitus rats. Pharm Biol 55 2170-2177, 2017.

18. Zuo ML, Wang AP, Tian Y, Mao L, Song GL and Yang ZB Oxymatrine ameliorates insulin resistance in rats with type 2 diabetes by regulating the expression of KSRP, PETN, and AKT in the liver. J Cell Biochem 120: 16185-16194, 2019.

19. Yoshioka K, Saito M, Oh KB, Nemoto Y, Matsuoka H, Natsume $\mathrm{M}$ and Abe $\mathrm{H}$ : Intracellular fate of 2-NBDG, a fluorescent probe for glucose uptake activity, in Escherichia coli cells. Biosci Biotechnol Biochem 60: 1899-1901, 1996.

20. Livak KJ and Schmittgen TD: Analysis of relative gene expression data using real-time quantitative PCR and the 2(-Delta Delta C(T)) method. Methods 25: 402-408, 2001.

21. Zdychova J and Komers R: Emerging role of AKT kinase/protein kinase B signaling in pathophysiology of diabetes and its complications. Physiol Res 54: 1-16, 2005.

22. Huang X, Liu G, Guo J and Su Z: The PI3K/AKT pathway in obesity and type 2 diabetes. Int J Biol Sci 14: 1483-1496, 2018.

23. Vinayagam A, Kulkarni MM, Sopko R, Sun X, Hu Y, Nand A, Villalta C, Moghimi A, Yang X, Mohr SE, et al: An integrative analysis of the InR/PI3K/AKT network identifies the dynamic response to insulin signaling. Cell Rep 16: 3062-3074, 2016.

24. Bathina S and Das UN: Dysregulation of PI3K-AKT-mTOR pathway in brain of streptozotocin-induced type 2 diabetes mellitus in Wistar rats. Lipids Health Dis 17: 168, 2018.

25. Burgess SC, Hausler N, Merritt M, Jeffrey FM, Storey C, Milde A, Koshy S, Lindner J, Magnuson MA, Malloy CR and Sherry AD: Impaired tricarboxylic acid cycle activity in mouse livers lacking cytosolic phosphoenolpyruvate carboxykinase. J Biol Chem 279: 48941-48949, 2004.

26. Mutel E, Abdul-Wahed A, Ramamonjisoa N, Stefanutti A, Houberdon I, Cavassila S, Pilleul F, Beuf O, Gautier-Stein A, Penhoat A, et al: Targeted deletion of liver glucose- 6 phosphatase mimics glycogen storage disease type 1 a including development of multiple adenomas. J Hepatol 54: 529-537, 2011.
27. Jiang WJ, Wang S, Xiao M, Lin Y, Zhou L, Lei Q, Xiong Y, Guan KL and Zhao S: Acetylation regulates gluconeogenesis by promoting PEPCK1 degradation via recruiting the UBR5 ubiquitin ligase. Mol Cell 43: 33-44, 2011.

28. Koo SH, Flechner L, Qi L, Zhang X, Screaton RA, Jeffries S, Hedrick S, Xu W, Boussouar F, Brindle P, et al: The CREB coactivator TORC2 is a key regulator of fasting glucose metabolism. Nature 437: 1109-1111, 2005.

29. Matsumoto M, Pocai A, Rossetti L, Depinho RA and Accili D: Impaired regulmion of hepatic glucose production in mice lacking the forkhead transcription factor Foxol in liver. Cell Metab 6: 208-216, 2007.

30. Zhou XY, Shibusawa N, Naik K, Porras D, Temple K, Ou H, Kaihara K, Roe MW, Brady MJ and Wondisford FE: Insulin regulation of hepatic gluconeogenesis through phosphorylation of CREB-binding protein. Nat Med 10: 633-637, 2004.

31. Erion DM, Ignatova ID, Yonemitsu S, Nagai Y Chatterjee $P$, Weismann D, Hsiao JJ, Zhang D, 1wasaki T, Stark R, et al: Prevention of hepatic steatosis and hepatic insulin resistance by knockdown of cAMP response element-binding protein. Cell Metab 10: 499-506, 2009.

32. Cypess AM, Zhang H, Schulz TJ, Huang TL, Espinoza DO, Kristiansen K, Unterman TG and Tseng YH: Insulin/IGF-I regulation of necdin and brown adipocyte differentiation via CREB- and FoxO1-associated pathways. Endocrinology 152: 3680-3689, 2011.

33. Li X, Monks B, Ge Q and Birnbaum MJ: AKT/PKB regulates hepatic metabolism by directly inhibiting PGC-1alpha transcription coactivator. Nature 447: 1012-1016, 2007.

34. Abdul-Ghani MA, Puckett C, Triplitt C, Maggs D, Adams J, Cersosimo $\mathrm{E}$ and DeFronzo RA: Initial combination therapy with metformin, pioglitazone and exenatide is more effective than sequential add-on therapy in subjects with new-onset diabetes. Results from the efficacy and durability of initial combination therapy for Type 2 Diabetes (EDICT): A randomized trial. Diabetes Obes Metab 17: 268-275, 2015.

35. Guo C, Zhang C, Li L, Wang Z, Xiao W and Yang Z: Hypoglycemic and hypolipidemic effects of oxymatrine in high-fat diet and streptozotocin-induced diabetic rats. Phytomedicine 21: 807-814, 2014.

36. $\mathrm{Yu} \mathrm{XH,} \mathrm{Zhu} \mathrm{JS} \mathrm{and} \mathrm{Gu} \mathrm{GM:} \mathrm{Clinical} \mathrm{effect} \mathrm{of} \mathrm{oxymatrine}$ combined with metformin on insulin resistance and serum TNF- $\alpha$ in patients with non-alcoholic fatty liver. Chin J Clin Hepatol 23: 195-195, 2007.

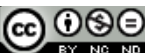

This work is licensed under a Creative Commons Attribution-NonCommercial-NoDerivatives 4.0 International (CC BY-NC-ND 4.0) License. 